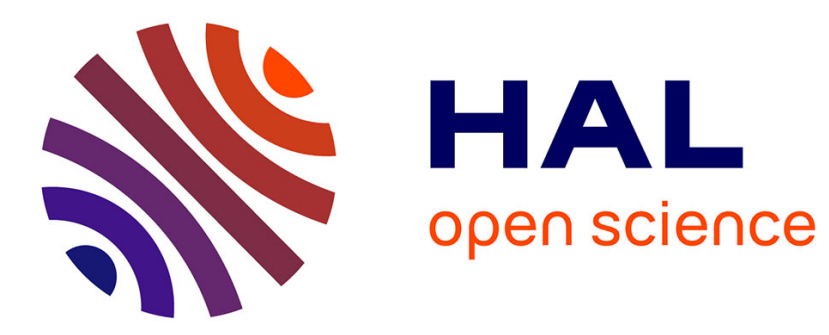

\title{
On the numerical modeling and optimization of a bottom-referenced heave-buoy array of wave energy converters
}

\author{
F Fàbregas Flavià, Aurélien Babarit, Alain H. Clément
}

\section{To cite this version:}

F Fàbregas Flavià, Aurélien Babarit, Alain H. Clément. On the numerical modeling and optimization of a bottom-referenced heave-buoy array of wave energy converters. International Journal of Marine Energy, 2017, 19, pp.1-15. 10.1016/j.ijome.2017.05.004 . hal-01531895

\section{HAL Id: hal-01531895 \\ https://hal.science/hal-01531895}

Submitted on 15 Nov 2017

HAL is a multi-disciplinary open access archive for the deposit and dissemination of scientific research documents, whether they are published or not. The documents may come from teaching and research institutions in France or abroad, or from public or private research centers.
L'archive ouverte pluridisciplinaire HAL, est destinée au dépôt et à la diffusion de documents scientifiques de niveau recherche, publiés ou non, émanant des établissements d'enseignement et de recherche français ou étrangers, des laboratoires publics ou privés. 


\title{
On the numerical modeling and optimization of a bottom-referenced heave-buoy array of wave energy converters
}

\author{
F. Fàbregas Flaviàa, ${ }^{\mathrm{a}, *}$ A. Babarit ${ }^{\mathrm{a}}$, A. H. Clément $^{\mathrm{a}}$ \\ ${ }^{a}$ Ecole Centrale de Nantes, LHEEA, UMR CNRS 6598, Nantes (France)
}

\begin{abstract}
Compact arrays of small wave absorbers have been proposed as an advantageous solution for the extraction of wave energy when compared to a big isolated point absorber. Numerous challenges are associated with the numerical modeling of such devices, notably the computation of the hydrodynamic interactions among the large number of floats of which they are composed. Efficient calculation of the first-order linear hydrodynamic coefficients requires dedicated numerical tools, as their direct computation using standard boundary element method (BEM) solvers is precluded. In this paper, the Direct Matrix Method interaction theory by Kagemoto and Yue (1986) is used as an acceleration technique to evaluate the performance of a generic wave energy converter (WEC) inspired by the Wavestar $S C$-concept and to perform layout optimization. We show that there exists an optimum number of floats for a given device footprint. Exceeding this number results in a "saturation" of the power increase, which is undesirable for the economic viability of the device. As in previous studies on multiple absorber WECs, significant differences were observed in energy production among floats, due to hydrodynamic interactions.

Keywords: Multi-body WEC, Interaction Theory, Boundary Element Method, Optimization
\end{abstract}

\footnotetext{
* Corresponding author

Email address: francesc.fabregas-flavia@ec-nantes.fr (F. Fàbregas Flavià)
} 


\section{Introduction}

A great variety of technologies to extract power from ocean waves have been proposed, some of which are currently under development. These wave energy converters (WECs) may be classified by several methods (Falcão, 2010), for ex-

5 ample on the basis of size: devices whose characteristic length is much smaller than the wave-length of the incoming waves are referred to as point absorbers, and have been the object of numerous studies. Their responses are characterized by a resonant peak over a narrow band of frequencies of the incident wave spectra, and control strategies may be applied to increase their energy absorption (Falnes, 2001).

Another category, often referred to as multi-body WECs, consists of a group of multiple closely-spaced point absorbers attached to a common fixed or floating support structure. Within this category, several configurations have been proposed, including the $\mathrm{FO}^{3}$ platform (Taghipour and Moan, 2008), the Manchester Bobber (Weller et al. 2010) and the Wavestar (Hansen and Kramer, 2011). The former two consist of a square lattice of floats linked to a common supporting structure through a Power Take-Off (PTO) system. In contrast, floats in the latter are distributed with a linear arrangement and connected to both sides of a fixed bridge structure through rigid arms.

Inspired by the $\mathrm{FO}^{3}$ device, Garnaud and Mei (2009) analyzed the performance of compact square and circular arrays of cylindrical point absorbers and compared them to a bigger float having an equivalent displacement. They found that, unlike the large buoy, the circular array of multiple point absorbers had good efficiency over a broad range of frequencies. They made use of the method of homogenization, which offers great savings in computational time, and is valid when both the device size and the separating distance between units is small in comparison to the incident wave length.

A different acceleration technique, a mode expansion method (Newman 1994), was used by Taghipour and Moan (2008) to study the $F O^{3}$ device. They so evaluated both the response of the floating rig supporting 21 floats and the 
wave energy absorption capabilities of the WEC. For this particular configuration, they found that the power produced was independent of the mean wave direction for short-crested ocean waves. In addition, they observed significant differences in power production between floats.

A comparison of two $F O^{3}$-type WECs, one with 21 aligned buoys and the other with a staggered grid configuration of twelve buoys, was performed by De Backer et al. (2010). Calculations were undertaken in the frequency domain and the hydrodynamic coefficients were calculated using the Boundary Element Method (BEM) code WAMIT. They observed that the 21-unit configuration 40 was able to produce only $25 \%$ more power than the 12 -unit configuration. A similar result was observed in experiments carried out by Garnaud and Mei (2009) in which an increase in the density of floats for tight configurations ded to a relatively small increase in capture width. The work of De Backer et al. (2010) addressed the impact of constraints and several PTO optimization

45 strategies. It was found that the former reduced the power production of the arrays whereas the application of individual optimization led to a significant increase in energy capture when compared to other less sophisticated strategies. The same conclusion was reached by Nambiar et al. (2015) after a study of three buoys of the Wavestar prototype that compared different types of resistive and reactive PTO control strategies using a dedicated time domain model including PTO damping force constraints.

Different versions of the Wavestar multi-body WEC device have been presented in Hansen et al. (2013). In the present paper, as we wish to illustrate the interest of the Direct Matrix Method when dealing with large groups of floating bodies, we choose the 60 -float $S C$-concept as a working example. The objectives are i) to examine the power capture of a generic bottom-referenced heave-buoy array (BR-HBA) inspired by this WEC, and ii) to conduct an optimization of both its layout and the size of the floats.

The study is carried out in the frequency domain using linear potential flow 60 theory. No constraints nor sophisticated Power Take-Off tuning strategies have been considered herein and, as in De Backer et al. (2010), the effect of diffracted 
waves from the supporting piles of the structure has not been addressed. Therefore, results should be regarded as preliminary estimates of the power generation potential of this type of technology.

In the following sections, a detailed description of the system is provided and the numerical modeling in the frequency domain is detailed, with particular emphasis on the procedure used for efficient computation of the hydrodynamic coefficients of the floats in the array. Some results are then presented, detailing the response of both an individual and a small cluster of three floats. Following the analysis of individual units, relevant layout configurations derived from optimization studies on the reference 60-unit configuration are analyzed in detail. Finally, results concerning the impact of float size on power capture are presented.

\section{Methodology}

75 2.1. Description of the System

The bottom-referenced heave-buoy array WEC studied herein is composed of 60 hemispherical floats regularly distributed along both sides of each of the three arms of a fixed bridge structure (see Figure 1). Each individual float is rigidly connected to an arm mounted on the supporting frame by means of

so a hinge joint. In our modeling, the hydraulic Power Take-Off (PTO), which transforms the rotation into electrical power in the real device, is replaced by a basic linear damper.

A global Cartesian reference system $(X, Y, Z)$ is used to define the ambient incident wave propagation angle $(\beta)$ with respect to the multi-body WEC. In addition, a local Cartesian reference system $(x, y, z)$ centered at each float is used to redefine the incident wave angle with respect to each individual unit. Figure 1 shows a schematic of the system, and the main parameters are specified in Table 1 . 

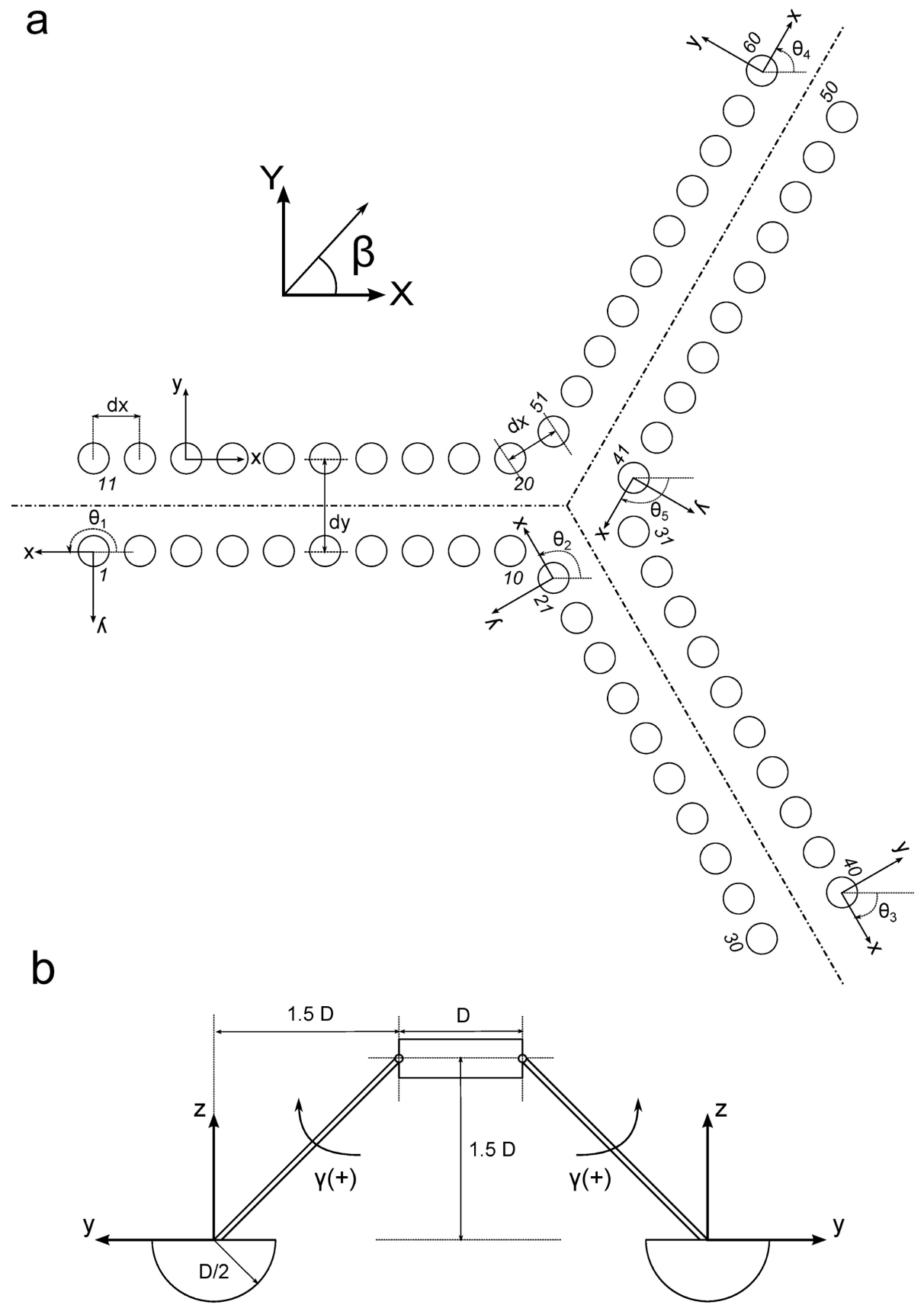

Figure 1: Top (a) and Side (b) schematic views of the bottom-fixed heave-buoy array Wave Energy Converter. 


\begin{tabular}{cc} 
Parameter & Value \\
\hline$d x$ & $7.2 \mathrm{~m}$ \\
$d y$ & $24 \mathrm{~m}$ \\
$D$ & $6 \mathrm{~m}$ \\
$\gamma_{\max }$ & $0.28 \mathrm{rad}$ \\
$\theta_{1}$ & $\pi \mathrm{rad}$ \\
$\theta_{2}$ & $2 \pi / 3 \mathrm{rad}$ \\
$\theta_{3}$ & $-\pi / 3 \mathrm{rad}$ \\
$\theta_{4}$ & $\pi / 3 \mathrm{rad}$ \\
$\theta_{5}$ & $-2 \pi / 3 \mathrm{rad}$ \\
\hline
\end{tabular}

Table 1: Main parameters of the bottom-fixed heave-buoy array Wave Energy Converter.

\subsection{Equation of Motion}

The linear first-order equation of motion of a single hemispherical point absorber float can be written as:

$$
(J+A) \ddot{\gamma}+\left(B+B_{p t o}\right) \dot{\gamma}+K^{h} \gamma=M^{e x}
$$

90

where $\gamma$ is the angle of rotation along the bearing axis, $J$ the inertia of the float, $A$ and $B$ the radiation hydrodynamic coefficients of added-inertia and damping moment respectively, $B_{p t o}$ the damping moment of the PTO system, $K^{h}$ the hydrostatic stiffness and $M^{e x}$ the excitation moment.

Assuming that the rigid arm connecting the float to the bearing is weightless, the hydrostatic stiffness coefficient $K^{h}$ expressed with respect to the axis of rotation can be computed as (Babarit et al., 2012):

$$
K^{h}=K_{B}^{h, \text { roll }}+\rho g V\left(z_{B}-z_{A}\right)-m g\left(z_{G}-z_{A}\right)+K_{B}^{h, \text { heave }}\left(y_{B}-y_{A}\right)^{2}
$$

where $K_{B}^{h, \text { roll }}$ and $K_{B}^{h, \text { heave }}$ are the hydrostatic stiffness related to the roll and heave motions, respectively, along the axis passing through the center of buoyancy of the float, $\rho$ the water density, $g$ the gravity acceleration, $V$ the volume of the float, $\left(x_{B}, y_{B}, z_{B}\right)$ the coordinates of the center of buoyancy, $\left(x_{G}, y_{G}, z_{G}\right)$ 


\begin{tabular}{ccl} 
Parameter & Expression & Value \\
\hline$V$ & $2 / 3 \pi \frac{D^{3}}{8}$ & $56.55 \mathrm{~m}^{3}$ \\
$K_{B}^{h, \text { heave }}$ & $\rho g \pi \frac{D^{2}}{4}$ & $284305 \mathrm{Kg} \cdot \mathrm{m} / \mathrm{s}^{2}$ \\
$K_{B}^{h, \text { roll }}$ & $\rho g \pi 6 / 24 \frac{R^{4}}{16}$ & $639687 \mathrm{Kg} \cdot \mathrm{m} / \mathrm{s}^{2}$ \\
$\left(x^{G}, y^{G}, z^{G}\right)$ & $\left(0,0,-\frac{3}{8} \frac{D}{2}\right)$ & $(0,0,-1.125) \mathrm{m}$ \\
$\left(x^{B}, y^{B}, z^{B}\right)$ & $\left(0,0,-\frac{3}{8} \frac{D}{2}\right)$ & $(0,0,-1.125) \mathrm{m}$ \\
$\left(x^{A}, y^{A}, z^{A}\right)$ & $(0,-1.5 D, 1.5 D)$ & $(0,-9,9) \mathrm{m}$ \\
$J$ & $\frac{83}{320} \rho V \frac{D^{2}}{4}+\rho V d^{2}$ & $1.08 \cdot 10^{7} \mathrm{Kg} \cdot \mathrm{m}^{2}$ \\
$d$ & $\sqrt{(1.5 D)^{2}+\left(1.5 D+\frac{3}{8} \frac{D}{2}\right)^{2}}$ & $13.55 \mathrm{~m}$ \\
\hline
\end{tabular}

Table 2: Main parameters of an hemispheric float.

the coordinates of the center of gravity and $\left(x_{A}, y_{A}, z_{A}\right)$ the coordinates of the bearing. Table 2 summarizes the values of the parameters for an hemispheric float of the configuration shown in Figure 1

Equation (1) can be generalized to include the motion of all the multi-body WEC floats:

$$
(\mathbf{J}+\mathbf{A}) \ddot{\Gamma}+\left(\mathbf{B}+\mathbf{B}_{p t o}\right) \dot{\Gamma}+\mathbf{K}^{h} \Gamma=\mathbf{M}^{e x}
$$

where $\Gamma$ is the vector of rotations, $\mathbf{J}$ is the diagonal inertia matrix of the system, $\mathbf{A}$ and $\mathbf{B}$ are the matrices of hydrodynamic added-inertia and radiation damping moment coefficients respectively, $\mathbf{B}_{p t o}$ is the diagonal matrix of PTO damping moments, $\mathbf{K}^{h}$ is the matrix of hydrostatic stiffness and $\mathbf{M}^{e x}$ the vector of excitation moments. All the matrices have dimensions $\left(N_{b} \times N_{b}\right)$, where $N_{b}$ is the total number of floats.

\subsection{Computation of the Hydrodynamic Coefficients}

The use of standard Boundary Element Method (BEM) solvers to evaluate the hydrodynamic coefficient matrices for large arrays of bodies, such as the bottom-referenced heave-buoy array WEC, is associated with very high computational costs. This hampers analysis of such systems and precludes the use 
of layout optimization, which requires constant recomputation of the hydrodynamic coefficients to account for modifications to the position of the floats.

To avoid the limitations imposed by the use of standard BEM solvers in the current study, the hydrodynamic coefficients were evaluated using the implementation of the Direct Matrix Method Interaction Theory (IT) by Kagemoto and Yue (1986), described in Fàbregas Flavià et al. (submitted for publication). As opposed to standard BEM solvers, in which the multiple-scattering problem is solved by treating all the bodies in the array simultaneously, in the Direct Matrix Method the diffraction/radiation boundary conditions are first imposed on an isolated float and then combined with an interaction theory to take into account the effect of the neighbouring devices. As in the present case, where all the floats have the same geometry, the boundary value problem (BVP) needs to be solved only once, thus leading to an additional gain in computational speed.

The transfer from the diffraction/radiation problem of an isolated geometry to the BVP of the whole array is achieved by expressing the wave fields in a base of partial cylindrical wave functions. This enables the waves diffracted and radiated by a float to be mathematically characterized in terms of two hydrodynamic operators known as Diffraction Transfer Matrix (DTM) and Radiation Characteristics(RC). The advantage of this transformation is that the number of cylindrical modes required to represent the perturbation of the wave field by an arbitrary geometry is significantly smaller than the number of panels required to discretize its wetted surface. The reduction in the number of unknowns associated to an individual float enables a drastic reduction of the computational time for the whole array, as shown in section 3.4 .

\subsection{Performance evaluation}

The total average annual power generated by a float $j$ in the multi-body WEC $\left(P_{y}^{j}\right)$ can be computed by summing up the contribution from each of the wave climate sea states as:

$$
P_{y}^{j}=\sum_{i=1}^{N_{s}} O_{i}\left(H_{s}, T_{p}\right) \cdot P_{i}^{j}\left(H_{s}, T_{p}\right)
$$


where $P_{y}^{j}$ is the total average annual power produced by float $j, N_{s}$ is the number of sea states considered, $\left(H_{s}, T_{p}\right)$ are the significant wave height and the peak period of the sea state, $O_{i}$ its probability of occurrence and $P_{i}^{j}$ the 140 power produced by unit $j$ in the $i$ th sea state.

Under the assumptions of linear potential flow theory, i.e. linearity and ideal fluid characteristics, the power generated by a float in a given sea state can be evaluated using the following expression:

$$
P_{i}^{j}\left(H_{s}, T_{p}\right)=\int_{0}^{2 \pi} \int_{0}^{\infty} 2 S_{i}(\omega, \beta) p^{j}(\omega, \beta) d \omega d \beta
$$

where $S_{i}(\omega, \theta)$ is the directional wave spectrum and $p^{j}(\omega, \theta)$ the power function of body $j$ defined as:

$$
p^{j}(\omega, \beta)=\frac{1}{2} B_{p t o} \omega^{2} \Gamma^{j}(\omega, \beta)^{2}
$$

where $B_{\text {pto }}$ is the Power Take-Off (PTO) damping and $\Gamma^{j}(\omega, \beta)$ is the response amplitude operator (RAO) of the productive degree of freedom of the hemispheric float obtained by solving the equation of motion of the system (3).

The total average annual power $\left(P_{y}\right)$ produced by all units is obtained simply as:

$$
P_{y}=\sum_{j=1}^{N_{b}} P_{i}^{j}\left(H_{s}, T_{p}\right)
$$

The effect of the hydrodynamic interactions among floats in the multi-body WEC on the power generation is quantified using the interaction factor, generally referred to as q-factor, defined as the ratio between the power produced by the float in the array and the power it would produce if isolated:

$$
q_{i}^{j}=\frac{P_{i}^{j}}{P_{i}^{j, i s o l}}
$$

where $q_{i}^{j}$ is the interaction factor of float $j$ in the $i$ th sea state and $P_{i}^{j, i s o l}$ the power that an isolated float $j$ would produce in the $i$ th sea state.

To evaluate whether the constraint on the rotation angle of each unit is satisfied, the significant amplitude of rotation of float $j, \gamma_{s}^{j}$, can be computed 
as (De Backer et al., 2010):

$$
\gamma_{s}=2 \sqrt{\int_{0}^{\infty} \int_{0}^{2 \pi} S_{\gamma}^{j}(f, \beta) d f d \beta}
$$

150

where $S_{\gamma}^{j}(f, \beta)$ is the rotation spectrum of float $j$ evaluated as:

$$
S_{\gamma}^{j}(f, \beta)=\frac{\Gamma^{j}(f, \beta)^{2}}{2 d f d \beta}
$$

\subsection{Wave Climate}

A total of eleven sea states (Table 3) representing $80 \%$ of the wave conditions at Hanstholm (Hansen and Kramer, 2011) were used for the computations. It was considered that the other $20 \%$ fall outside of the range of operation of the multi-body WEC, i.e. production is stopped when $H_{s}<0.75 \mathrm{~m}$ and protection mode is activated for cases with $H_{s}>2.75 \mathrm{~m}$.

The wave field was modeled as a two-dimensional frequency-direction Bretschneider spectrum with a generalized cosine angular spreading function $D(\beta)=$ $A_{1} \cos ^{m}(\beta-\bar{\beta})$ as defined in Holthuijsen (2010) with $m=20$. The latter is representative of wind seas predominant in the site. The spectrum was discretized using 30 frequencies and five directions making a total of 150 wave components per sea state. The water depth was set to $20 \mathrm{~m}$.

\section{Results and Discussion}

\subsection{Individual float response}

The solution to the equation of motion (1) of an individual articulated hemispherical float is shown in Figure 2 together with the power extracted, the latter computed using equation (6). The response of the float shows the typical features of a point absorber, namely a steady response at low frequencies, an increased motion at the resonant frequency which is greatly reduced when reaching the higher frequency zone. A particularity of the arm-float system studied herein is that, in spite of the axisymmetry of the float, its response is highly dependent on the incident angle of the waves. This is because the components 


\begin{tabular}{cccc} 
Seastate & $H_{s}(m)$ & $T_{p}(s)$ & $\operatorname{Prob}(\%)$ \\
\hline 1 & 0.75 & 4.10 & 19.2 \\
2 & 0.75 & 5.27 & 11.4 \\
3 & 0.75 & 6.44 & 2.21 \\
4 & 1.25 & 4.10 & 6.84 \\
5 & 1.25 & 5.27 & 13.0 \\
6 & 1.25 & 6.44 & 2.96 \\
7 & 1.75 & 5.27 & 9.58 \\
8 & 1.75 & 6.44 & 3.05 \\
9 & 2.25 & 5.27 & 3.34 \\
10 & 2.25 & 6.44 & 4.6 \\
11 & 2.75 & 6.44 & 3.89 \\
\hline
\end{tabular}

Table 3: Wave climate used in the simulations.

of the excitation force in both $y$ and $z$ directions contribute to the moment along the axis of rotation $(x)$. An incident wave with $\beta=90^{\circ}$ will generate $y$ and ${ }_{175} z$ force components which will produce rotation moments acting in the same direction. In contrast, at $\beta=270^{\circ}$ the rotation moments will act in opposite directions. From Figure 2 it can be observed that the maximum response is achieved for an incident wave angle of $\beta=90^{\circ}$, which corresponds to the situation where the moments of rotation generated by the forces in $y$ and $z$ direction are aligned. The incident angle for which the minimum response occurs also depends on the balance between the magnitudes of the $y$ and $z$ forces.

Both the power and the response of the float are shown for two different values of $B_{p t o}$, indicated as $B p t o_{c}$ and Bpto on the graphs. The former is the value that maximises the response allowed $\left(\gamma_{\max }\right)$ at the resonant frequency and 185 for an incident wave angle of $\beta=90^{\circ}$. The latter has been tuned to reduce the float motion to $20 \%$ of the critical value at the same conditions. 

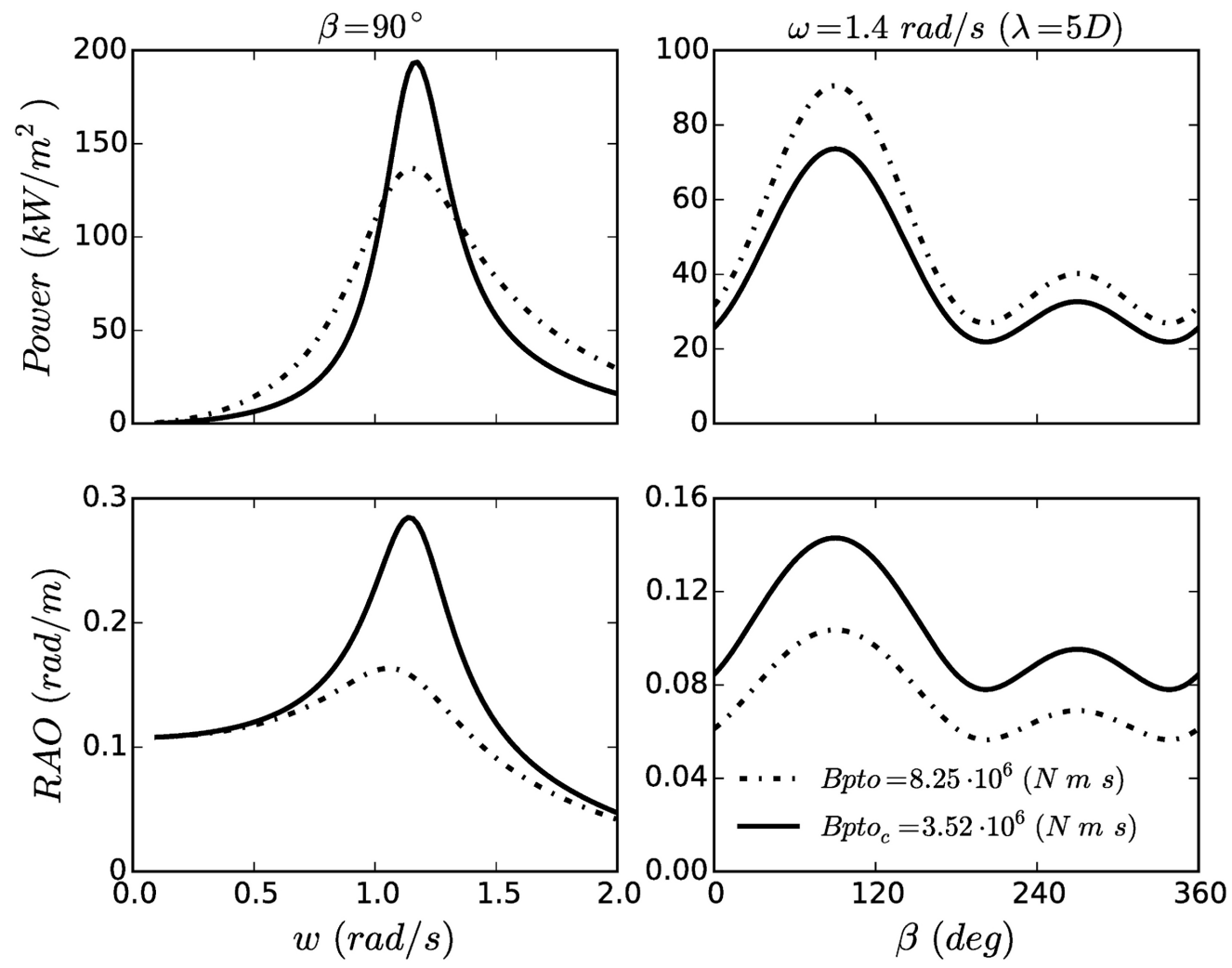

Figure 2: Response Amplitude Operator (RAO) and power function of an individual isolated hemispherical float as a function of the Power Take-Off damping $\left(B_{p t o}\right)$ and for both a range of incoming wave frequencies and directions. 


\subsection{Three-float cluster}

The presence of adjacent units affects the hydrodynamic forces acting on a float and, as a consequence, its response is different to that where it is in sensitivity studies were performed (detailed in section 3.3. 


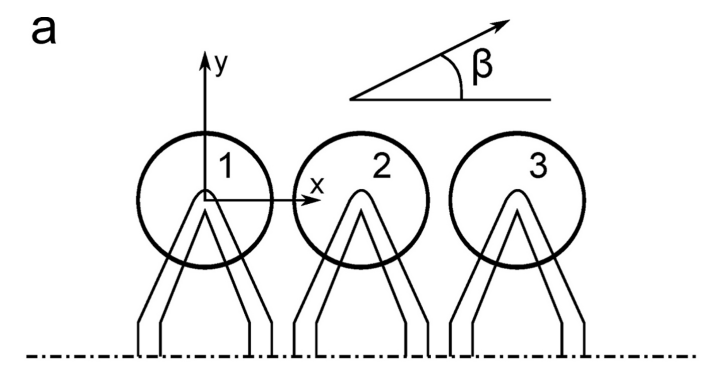

b

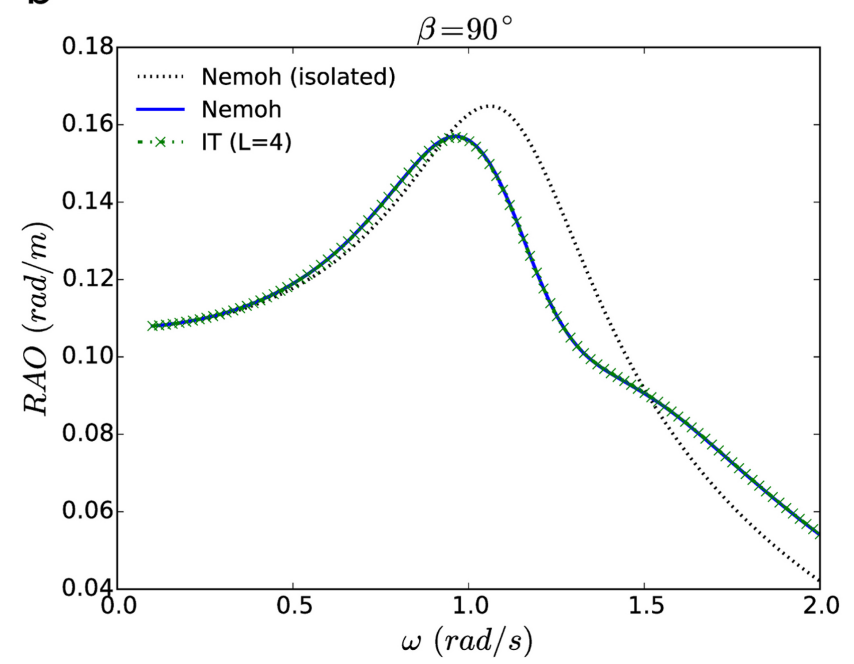

C

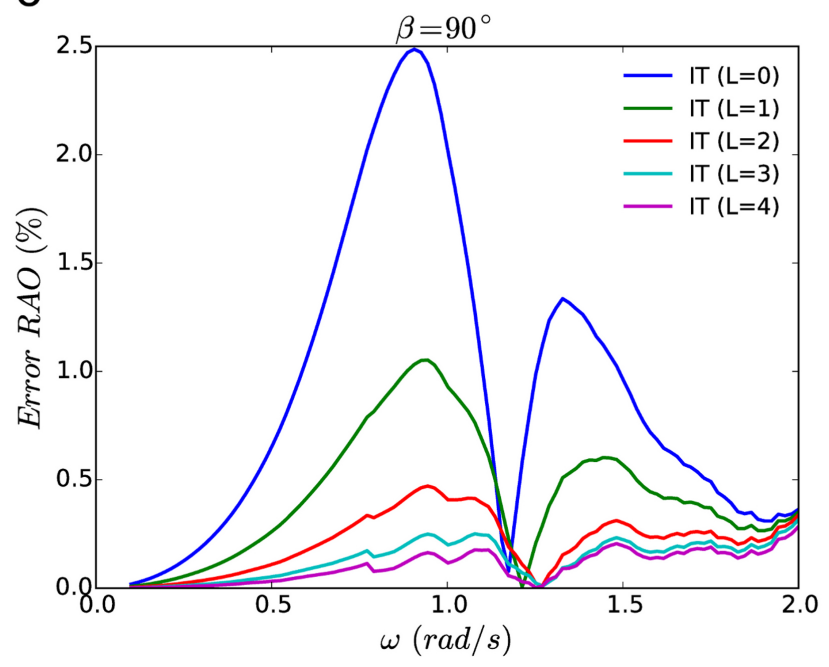

Figure 3: (a) Top view of a section of a three floats WEC; (b) Comparison of the Response Amplitude Operator (RAO) of float 2 computed using both a direct BEM calculation (both when isolated and in array) and with the Direct Matrix Method interaction theory $(I T)$ using 4 evanescent modes $(L)$ and a $\beta=90^{\circ}$ wave incidence; (c) relative difference between the (RAO) of float 2 computed using both a direct BEM calculation and with the Direct Matrix Method interaction theory $(I T)$ for different values of the evanescent modes truncation $(L)$ and a wave incidence of $\beta=90^{\circ}$. 


\subsection{0-float Configuration}

This section presents a series of simulations of the bottom-referenced heave$\beta=\pi / 3)$ of the incident wave spectrum. For the former, it can be observed that the interaction factor of the front floats $\left(f_{1}\right.$ and $\left.f_{11}\right)$ is significantly greater than 1 and gradually decreases as waves travel along the units in branches $b_{1}^{10}$ and $b_{11}^{20}$. At floats $f_{10}$ and $f_{20}$, behind the wake produced by the preceding units, most factor is only observed when moving away from the horizontal axis of symmetry of the WEC and towards the end of branches $b_{51}^{60}$ and $b_{21}^{30}$. Notwithstanding, 
the values remain significantly lower than 1 . It is in this part of the domain where the highest disturbance coefficients are observed. In contrast, the wake behind the floats significantly affects the units at branches $b_{31}^{40}$ and $b_{41}^{50}$, which have interaction factors close to zero.

For $\beta=\pi / 3$ it can be observed that floats in branches $b_{1}^{10}$ and $b_{21}^{30}$ benefit from higher interaction factors than elsewhere, although they do not exceed unity. In addition, a region with high disturbance coefficients can be clearly distinguished in front of the device, as opposed to the wake area behind characterized by a reduced wave elevation. Only floats $f_{11}-f_{12}$ and $f_{39}-f_{40}$ are not shadowed by the units in the front branches for this particular alignment of the incident wave field and the WEC.

The pattern of interaction factor among the floats is similar for all sea states, with the only significant difference being an increase in the power produced by the units in branches $b_{11}^{20}$ and $b_{31}^{40}$ for wave conditions with longer peak periods propagating with a main direction $\beta=\pi / 3$. As the $B_{\text {pto }}$ damping moment (identically applied to all units) was optimized to maximize the average annual power extracted, the interaction factors and the significant motions are closely related. From the left column of Figure 7, which shows the significant motions of each float for the most energetic sea state $\left(H_{s}=2.75 m, T_{p}=6.44 s\right)$, it can be noted that floats in branches $b_{11}^{20}-b_{31}^{40}$ undergo motions of the same order of magnitude as the ones in branches $b_{1}^{10}-b_{21}^{30}$. In contrast, for $\beta=0$, the pattern observed is similar to that for the interaction factors shown in Figure 4 Assuming the motions of the floats are governed by a Rayleigh distribution, as used in De Backer et al. (2010), and assuming the maximum significant motion to be $0.17 \mathrm{rad}$, it can be predicted that the design condition $\gamma_{\max }$ prescribed in Table 1 will be exceeded $\sim 25 \%$ of the time when the WEC operates in the most energetic sea state. This point is further investigated in section 3.6.

The main findings from a sensitivity study of the impact of the evanescent mode truncation on the average annual power production of the bottomreferenced heave-buoy array WEC are summarized in Table 4 . The greatest discrepancy in power extracted from a sea state, when computed using 2 evanes- 

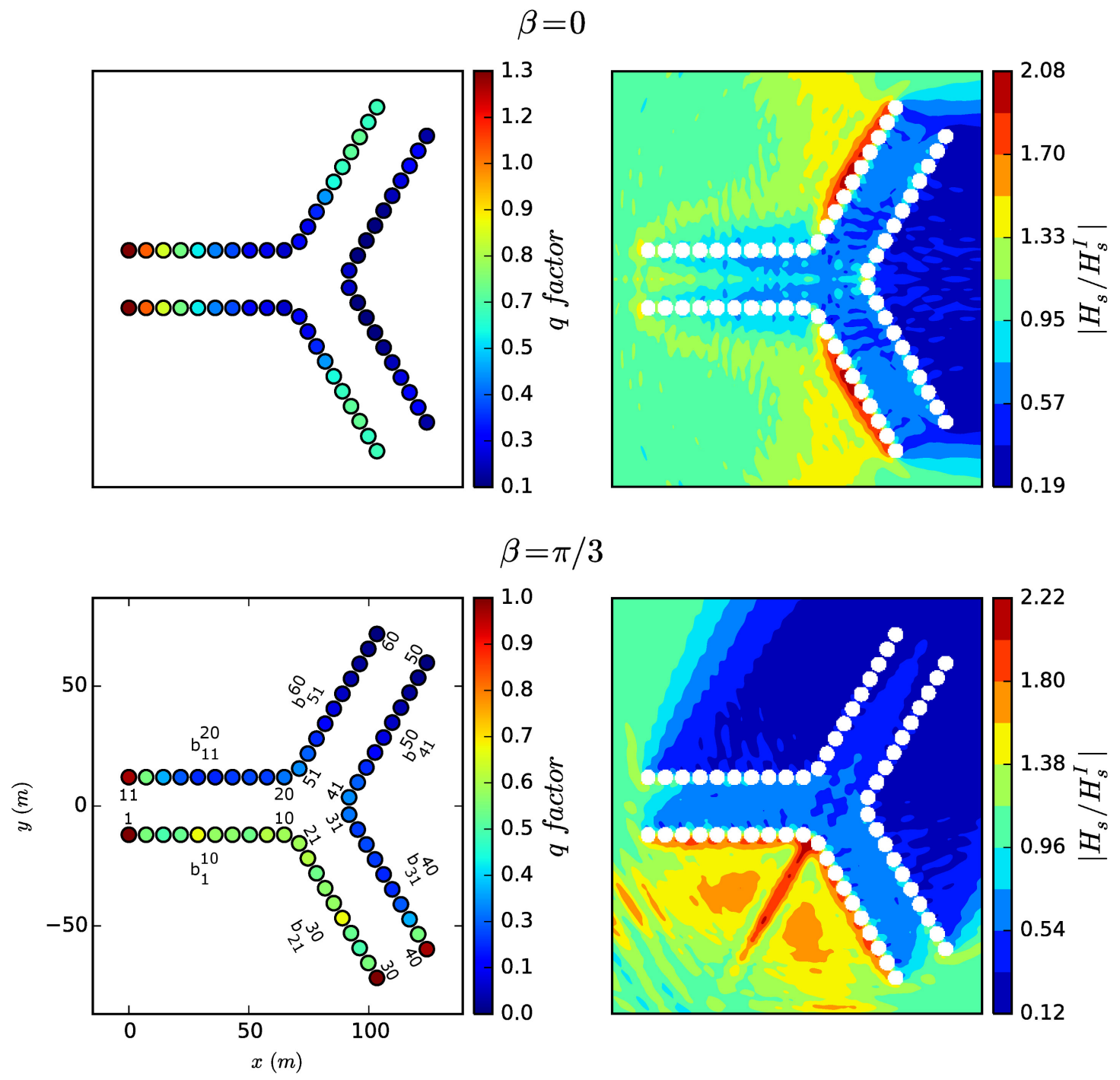

Figure 4: Float interaction factors (q-factor) of the bottom-referenced heave-buoy array WEC and disturbance coefficient $\left(H_{s} / H_{s}^{I}\right)$ of the wave field for a sea state with $\left(H_{s}=0.75 m\right.$, $\left.T_{p}=4 s\right)$ and two mean propagation directions $(0$ and $\pi / 3 \mathrm{rad})$ of the incident wave spectra. 


\begin{tabular}{|c|ccc|}
\hline Truncation $(n)$ & 0 & 1 & 2 \\
\hline $\max \left(\operatorname{abs}\left[\left(P_{n}^{i}-P_{2}^{i}\right) / P_{2}^{i}\right]\right)(\%)$ & 0.55 & 0.41 & Reference \\
\hline
\end{tabular}

Table 4: Influence of the evanescent modes truncation on the power generated.

280

Figure 5 shows the results of the optimization process, i.e. the average annual power produced by each configuration and the separating distance between units, for the two main propagation directions of the incident wave spec- 

in Figure 5 between the IT and standard direct NEMOH BEM calculations. Values are presented based on computations on a Dell ${ }^{1}$ machine with two Intel(R)

${ }^{1}$ Dell is a registered trademark of Dell, Inc.

tra. With the exception of the 12 -float configuration, average annual power is slightly greater when $\beta=\pi / 3$; for the 48 -float configuration, the difference is only $2.6 \%$. However, for both values of $\beta$ the change in average annual power as a function of number of floats $(\mathrm{N})$ follows similar trend: it increases linearly for $N \leq 30$; continues to increase nonlinearly until an inflection point is reached at $N=48$; then decreases towards the average annual power produced by the initial 60-float WEC configuration studied in the preceding section.

For both main propagation directions of the incident wave spectra $(\beta)$, the optimal separating distance for the 12 and 18-float configurations falls within the bounds imposed, although it is much closer to the upper limit than to the lower. This is also true for the 24 -float configuration when $\beta=0$. For all other cases, the optimal distance is the upper limit, which decreases as the number of units increases until merging with the separating distance $(7.2 \mathrm{~m})$ of the 60 -float WEC.

The above observations of average annual power and separating distance clearly show that increasing the number (and hence density) of floats in this particular three-arm structure layout has diminishing returns in terms of power production. Indeed, a remarkable result is that the same average annual power can be obtained with a 60 -float and 36-float configuration, the latter being studied in more detail in the following section. Obviously, this has dramatic implications with respect to the global cost of the WEC.

These results are in line with observations by De Backer et al. (2010) who showed differences of only $25 \%$ in average annual power production between two multi-body WECs of 21 and 12 floats. However, the size of the floats and their arrangement were not the same for both devices.

Table 5 compares the wall clock execution time required to obtain the results ues are presented based on computations on a Dell machine with two Intel(R) 

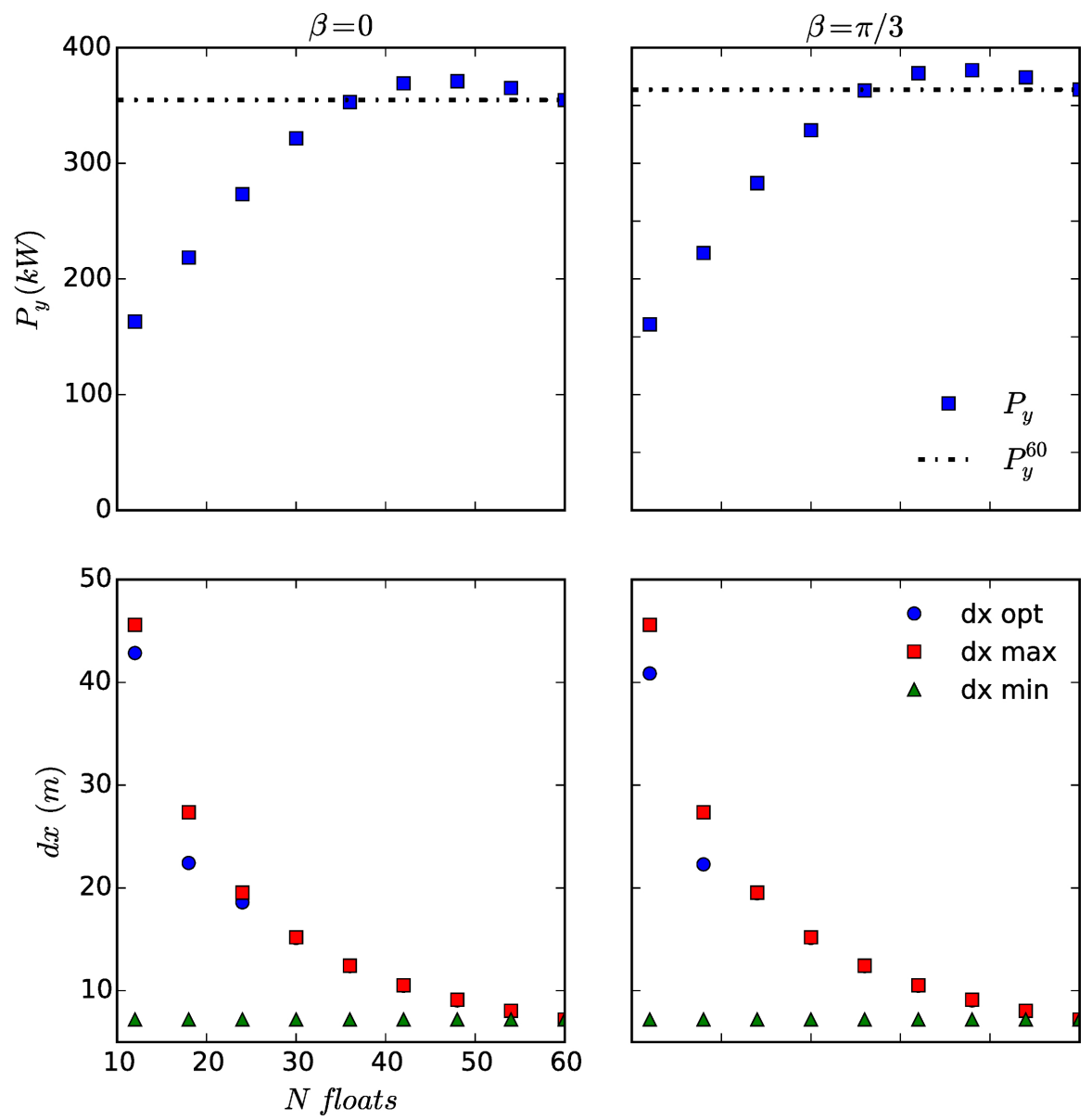

Figure 5: Average annual power $\left(P_{y}\right)$ and separating distance between floats $(d x)$ as a function of the number of units used in the bottom-referenced heave-buoy array WEC for two main propagation directions of the incident wave spectra. The average annual power has been computed using the optimized separating distance ( $d x o p t$ ) between floats for each configuration comprised between the limits $(d x \max )$ and $(d x \min )$. 


\begin{tabular}{cccc}
\hline N bodies & N function evaluations & IT (hours) & Direct BEM (years) \\
\hline 12 & 10 & 0.6 & 0.1 \\
18 & 10 & 1.3 & 0.3 \\
24 & 11 & 2.7 & 0.9 \\
30 & 10 & 3.7 & 1.6 \\
36 & 10 & 5.1 & 2.7 \\
42 & 9 & 6.4 & 3.8 \\
48 & 7 & 6.6 & 4.4 \\
54 & 6 & 7.3 & 5.4 \\
60 & 1 & 1.7 & 1.2 \\
\hline
\end{tabular}

Table 5: Comparison of wall clock execution time between the IT and direct NEMOH calculations for each of the optimized configurations in Figure 5

$\mathrm{Xeon}(\mathrm{R})^{2}$ 64-bit $2.27 \mathrm{GHz}$ processors and 8GB random access memory running the Microsoft Windows 7 Professional ${ }^{3}$ operating system. The execution time of the direct BEM simulations has been estimated based on a series of simulations on small configurations up to ten floats. The computational advantage of using the IT over standard BEM solvers to optimize the layout of large clusters of bodies is clear.

\subsection{6-float Configuration}

In this section, details of the 36-float bottom-referenced heave-buoy array WEC simulations are given. Figure 6 shows the interaction factor for each float and the free-surface disturbance coefficient for the most probable sea state $\left(H_{s}=0.75 m, T_{p}=4 s\right)$. Results are presented for the two main propagation directions $(\beta=0$ and $\pi / 3)$ of the incident wave spectrum considered. For the former, it can be observed that the interaction factor of floats $f_{1}$ and $f_{7}$ is significantly higher than unity and gradually decreases as waves propagate

\footnotetext{
${ }^{2}$ Intel Xeon is a registered trademark of Intel Corp.

${ }^{3}$ Microsoft Windows 7 Professional is a registered trademark of Microsoft Corp.
} 
along branches $b_{1}^{6}$ and $b_{7}^{12}$. At floats $f_{6}$ and $f_{12}$, shadowed by the units up front, most of the available energy has been absorbed and the values of the interaction factor are very low. The decreasing trend is reversed at branches $b_{31}^{36}$ and $b_{13}^{18}$, where the interaction factor gradually increases from low values at units $f_{13}$ and $f_{31}$ until reaching levels as high as at the front of branches $b_{1}^{6}$ and $b_{7}^{12}$ for floats $f_{29}$ and $f_{35}$. This differs from the 60 -unit configuration, whose floats at this part of the multi-body WEC had interaction factors significantly less than one (Figure 44. The wake behind branches $b_{1}^{6}$ and $b_{7}^{12}$, observed to be a region of low disturbance coefficient, diminishes the power production of floats in $b_{25}^{30}$ and $b_{19}^{24}$ close to the horizontal axis of symmetry of the device. However, it is higher here than for the 60-unit case. The interaction factor increases at the ends of branches $b_{25}^{30}$ and $b_{19}^{24}$, which benefit from a smaller shadowing effect.

For $\beta=\pi / 3$, similar trend is observed as in the 60 -float configuration, i.e. floats in branches $b_{1}^{6}$ and $b_{13}^{18}$ have interaction factors significantly higher than the rest. However, in this case they are all greater than unity. In addition, floats in branches $b_{7}^{12}$ and $b_{19}^{24}$ are less shadowed by units in $b_{1}^{6}$ and $b_{13}^{18}$. Disturbance coefficients close to unity are observed in this area due to both the reduced number of units and increased spacing between them. In contrast, an area of wave concentration can be distinguished in front of the device as opposed to a region of wave attenuation behind. The range of disturbance coefficients is significantly lower for the 36 -float configuration than for the 60 -unit tight cluster as the structure is more "transparent" to the incident waves.

As for the 60-float configuration, the pattern of interaction factors among the floats is similar for all sea states, with the only significant difference being an increase in power produced by the units in branches $b_{7}^{12}$ and $b_{19}^{24}$ for wave conditions with longer peak periods propagating with main direction $\beta=\pi / 3$. As mentioned in section 3.3 , the interaction factors and the significant motions are closely related. From the right column of Figure 7 which shows the significant motions of each float for the most energetic sea state $\left(H_{s}=2.75 \mathrm{~m}, T_{p}=6.44 \mathrm{~s}\right)$, it can be observed that the significant motions of floats in branches $b_{7}^{12}$ and $b_{19}^{24}$ are of the same order of magnitude as those in $b_{1}^{6}$ and $b_{13}^{18}$. In contrast, for $\beta=0$, 

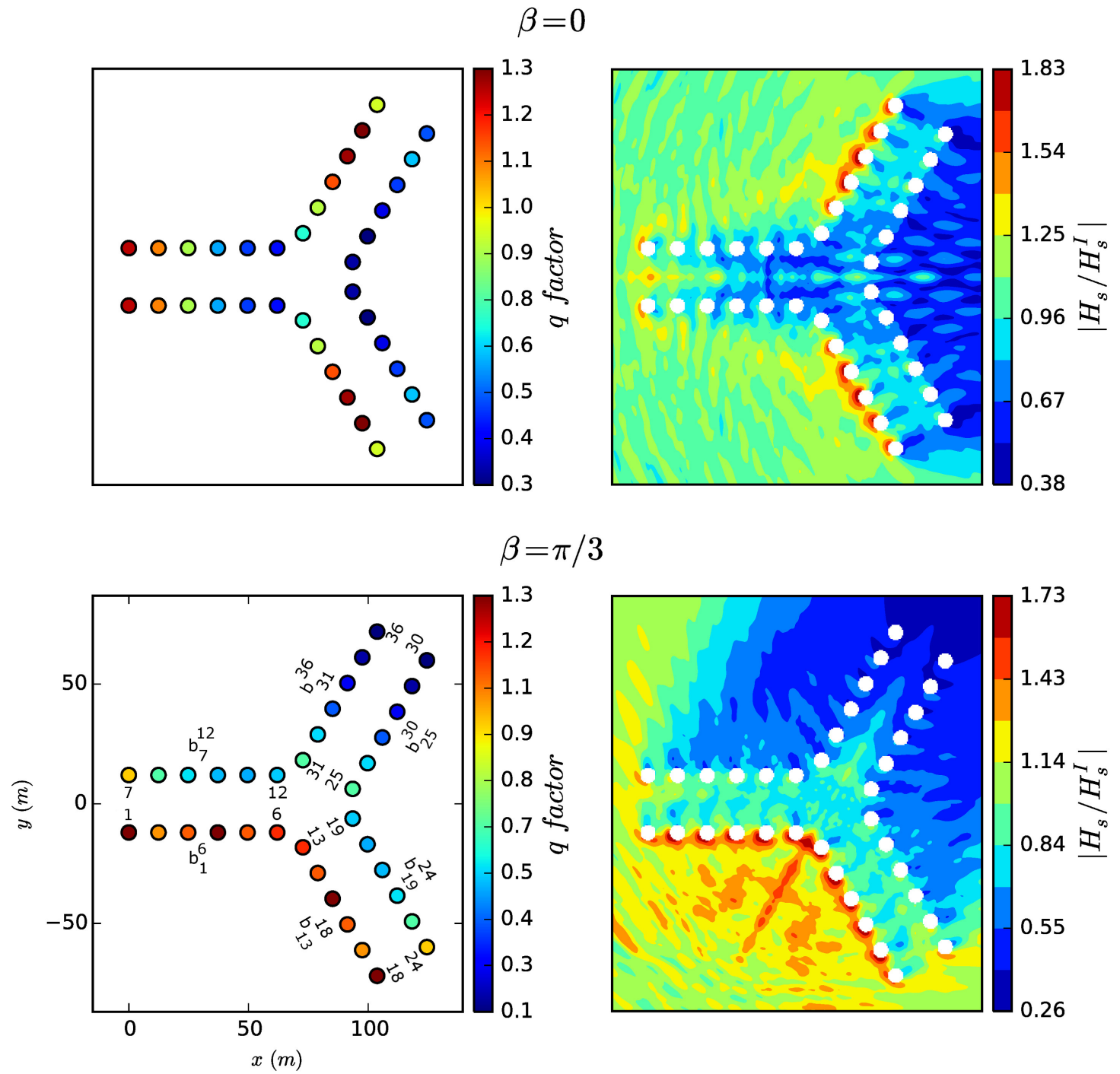

Figure 6: Float interaction factors (q-factor) of the 36-unit bottom-referenced heave-buoy array WEC and disturbance coefficient $\left(H_{s} / H_{s}^{I}\right)$ of the wave field for a sea state with $\left(H_{s}=\right.$ $\left.0.75 \mathrm{~m}, T_{p}=4 \mathrm{~s}\right)$ and two mean propagation directions $(0$ and $\pi / 3 \mathrm{rad})$ of the incident wave spectra. 
the pattern of significant motions is similar to that of the interaction factors.

For both the 60 -float and the 36-float configurations, the highest significant

Average annual power is observed to be a concave function of hemisphere radius, with a global maximum observed at $r=3.5 \mathrm{~m}$, for both values of $\beta$, 

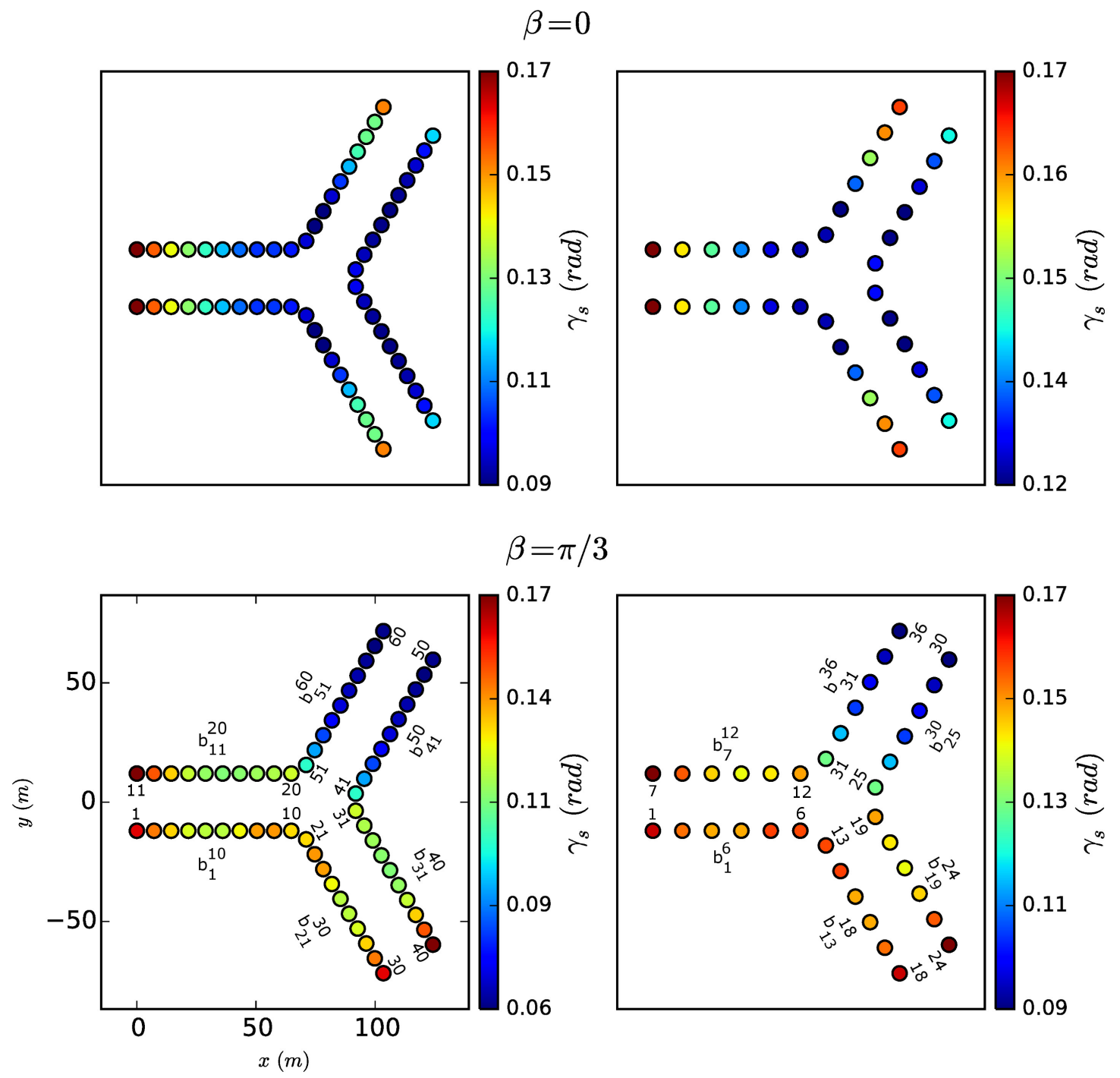

Figure 7: Float significant motions $\left(\gamma_{s}\right)$ of the 60 and 36-unit bottom-referenced heave-buoy array WEC for the sea state with $\left(H_{s}=2.75 m, T_{p}=6.44 s\right)$ and two mean propagation directions $(0$ and $\pi / 3 \mathrm{rad})$ of the incident wave spectra. 

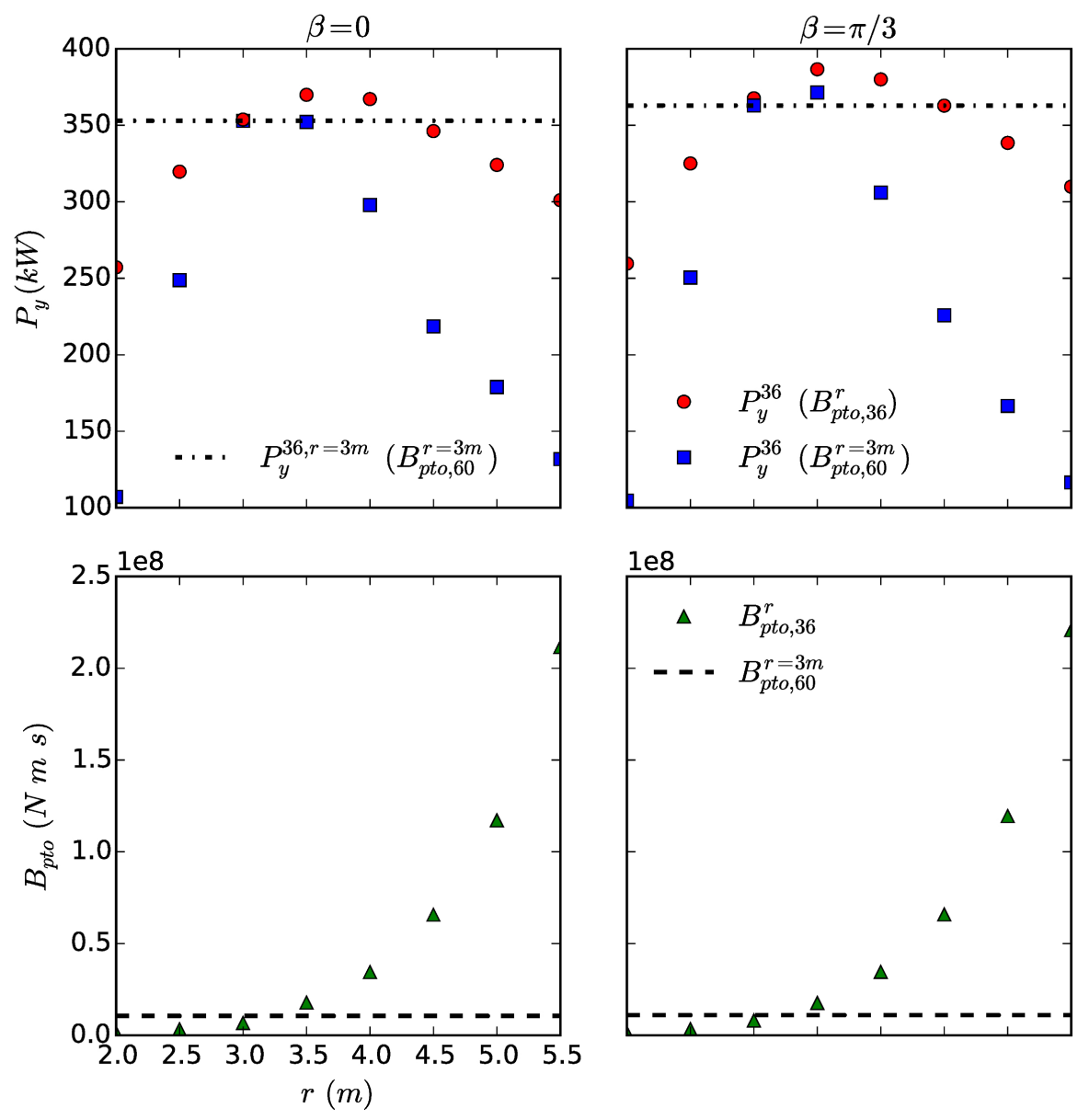

$1 e 8$

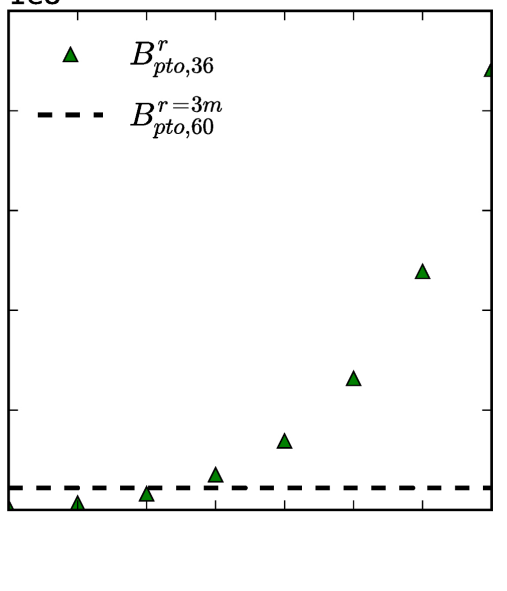

Figure 8: Average annual power $\left(P_{y}\right)$ produced by a 36-unit bottom-referenced heave-buoy array WEC as a function of both the radius of the hemispheric floats and the value of PTO damping $\left(B_{\text {pto }}\right)$ used for two main propagation directions of the incident wave spectra $(\beta)$. The values of PTO damping optimized for each float radius of the 36-unit configuration are indicated as $\left(B_{p t o, 36}^{r}\right)$ whereas $\left(B_{p t o, 60}^{r=3 m}\right)$ refers to the PTO damping which optimizes the average annual power produced by the 60 -unit configuration with $3 m$ radius floats. 
using the PTO damping moment $\left(B_{p t o, 36}^{r}\right)$ optimized for each float size. The value is $4.6 \%$ higher for $\beta=\pi / 3$ than for $\beta=0$. A remarkable result is that almost the same amount of power is produced for configurations with floats of radius $3 m$ or $4.5 m$, the difference between them being of only $2.3 \%$ and $1.4 \%$ for $\beta=0$ and $\pi / 3$ respectively. Using the PTO damping moment optimized for the 60-float configuration $\left(B_{p t o, 60}^{r=3 m}\right)$, a similar concave function is observed; however, the slope of the increasing and decreasing parts of the curve is significantly higher than for $\left(B_{p t o, 36}^{r}\right)$.

It is noteworthy that at a radius of $3 m$, for both main propagation directions $(\beta)$, no significant differences were observed between average annual power computed using the two different values of PTO damping. Using the fact that PTO damping moment is proportional to $r^{5}$, it can be inferred that the principal determinant of its optimization is not the number of floats but their size. When the PTO damping moment optimized for a radius of $3 m$ is applied $\left(B_{p t o, 60}^{r=3 m}\right)$, the average annual power produced in cases $r<3 m$ or $r>3 m$ deviates significantly from its maximum. The value of $B_{p t o}$ is too high and the float motions too small when $r<3 m$ and vice versa when $r>3 m$. This can be observed for the case $r=4.5 \mathrm{~m}$ in Figure 9, which shows the significant motions of each unit of the 36 -float configuration for both $\beta$ propagation directions. When $\left(B_{p t o, 60}^{r=3 m}\right)$ is used, float motions become significantly higher than when $\left(B_{p t o, 36}^{r=4.5 m}\right)$ is used. In the latter case, assuming the motions of the floats are governed by a Rayleigh distribution, and the maximum significant motion is $0.1 \mathrm{rad}$, it can be predicted that the design condition $\gamma_{\max }$ prescribed in Table1 1 will be exceeded only $\sim 2 \%$ of the time (for specific floats) when the WEC operates in the most energetic sea state. Although not shown here, for the same sea state the design condition is exceeded $\sim 20 \%$ of the time for the configuration with radius $3.5 \mathrm{~m}$ and its associated optimized $B_{p t o}$.

An economic assessment should be performed to identify the most advantageous design solution. On the one hand, despite satisfying the motion constraints imposed by the Power Take-Off configuration considered in this study, the use of units of radius $4.5 \mathrm{~m}$ would increase the total submerged volumne 
by $102 \%$ compared to the initial 60 -float WEC with hemispheres of radius $3 \mathrm{~m}$. duction, which in fact decreases such that the mean annual energy capture of 

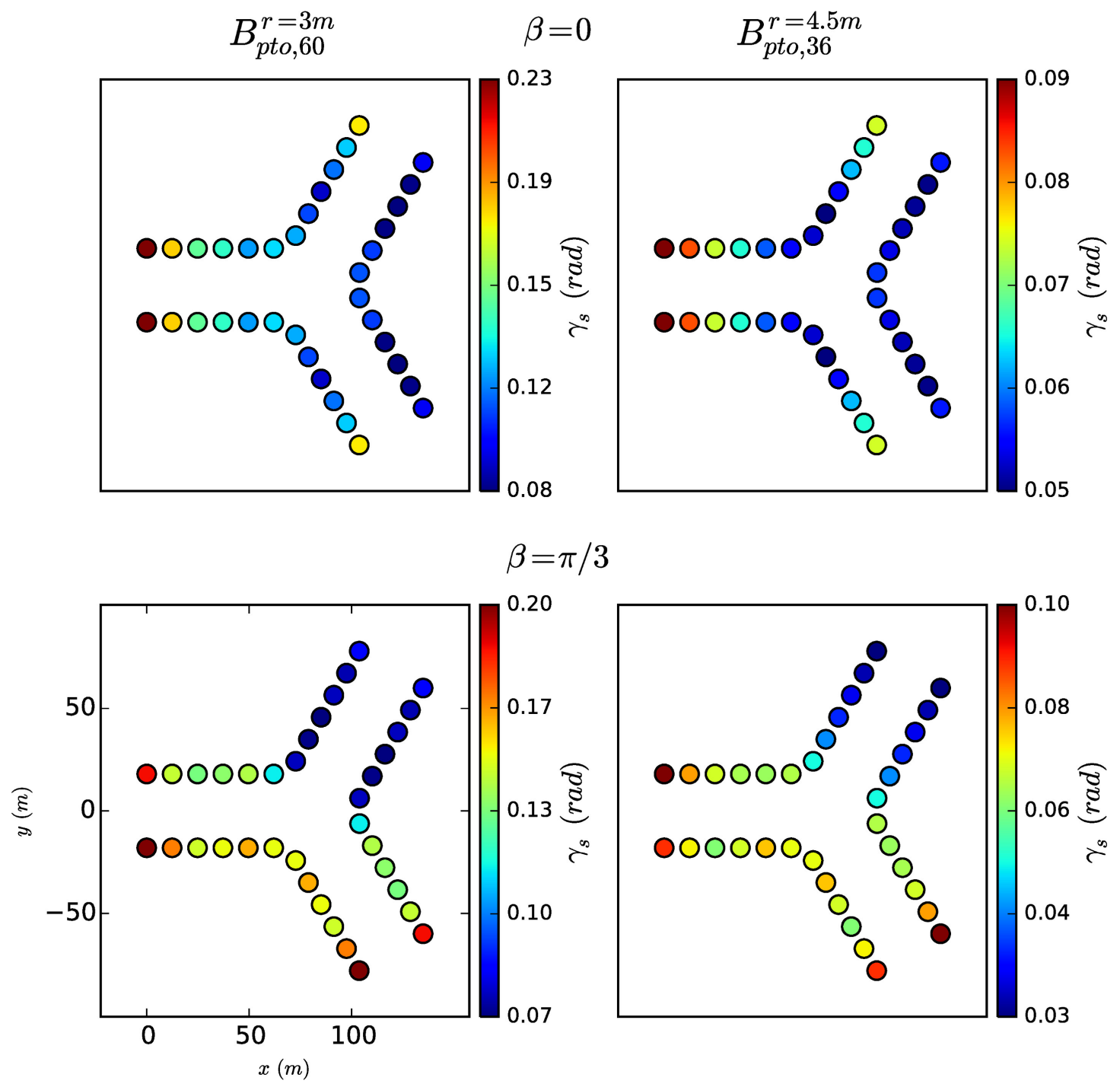

Figure 9: Float significant motions $\left(\gamma_{s}\right)$ of the 36-unit bottom-referenced heave-buoy array WEC with float radius $4.5 \mathrm{~m}$ for the sea state with $\left(H_{s}=2.75 \mathrm{~m}, T_{p}=6.44 \mathrm{~s}\right)$ and two mean propagation directions $(0$ and $\pi / 3 \mathrm{rad})$ of the incident wave spectra. Results are presented for two different values of PTO damping $\left(B_{p t o, 60}^{r=3 m}\right)$ and $\left(B_{p t o, 36}^{r=4.5 m}\right)$. The former is optimized to maximize the energy capture of a 60 -unit configuration with $3 \mathrm{~m}$ radius floats and the latter of a 36 -unit with $4.5 \mathrm{~m}$ radius floats. 
the 36 and the 60 -unit configurations are the same.

Finally, a sensitivity analysis was performed on the radius of the floats, from which it was found that optimal energy capture is achieved for a float radius of $3.5 \mathrm{~m}$.

NOTICE: the authors would like to mention that the data shown in the figures of this paper can be retrieved by downloading data files from the paper page on the editor internet site.

475 Acknowledgements

The research leading to these results is part of the OceaNET project, which has received funding from the European Union's Seventh Framework Programme for research, technological development and demonstration under Grant Agreement No. 607656.

\section{References}

Babarit A, Delhommeau G. Theoretical and numerical aspects of the open source BEM solver NEMOH. In: Proceedings of the 11th European Wave and Tidal Energy Conference 6-11th Sept 2015, Nantes, France. 2015. p. 112.

Babarit A, Hals J, Muliawan MJ, Kurniawan A, Moan T, Krokstad J. Numerical benchmarking study of a selection of wave energy converters. Renewable Energy 2012;41:44-63.

Chakrabarti S. Response due to moored multiple structure interaction. Marine Structures 2001;14(1-2):231-58. by closely spaced point absorbers in constrained conditions. IET Renewable Power Generation 2010;4(6):579. 
Fàbregas Flavià F, McNatt C, Rongère F, Babarit A, Clément AH. A numerical tool for the frequency domain simulation of large arrays of identical floating bodies. Ocean Engineering submitted for publication;.

Falcão AFdO. Wave energy utilization: A review of the technologies. Renewable and Sustainable Energy Reviews 2010;14(3):899-918.

Falnes J. Optimum control of oscillation of wave energy converters. International Journal of Offshore and Polar Engineering 2001;12.

Garnaud X, Mei CC. Wave-power extraction by a compact array of buoys. Journal of Fluid Mechanics 2009;635:389-413.

Hansen RH, Kramer MM. Modelling and Control of the Wavestar Prototype. Proceedings of the 9th European Wave and Tidal Energy Conference 2011;:110.

Hansen RH, Kramer MM, Vidal E. Discrete displacement hydraulic power takeoff system for the wavestar wave energy converter. Energies 2013;6(8):400144.

Holthuijsen LH. Waves in Oceanic and Coastal Waters. Cambridge University Press, 2010.

Kagemoto H, Yue DKP. Interactions among multiple three-dimensional bodies in water waves: an exact algebraic method. Journal of Fluid Mechanics 1986;166(-1):189.

Nambiar AJ, Forehand DIM, Kramer MM, Hansen RH, Ingram DM. Effects of hydrodynamic interactions and control within a point absorber array on electrical output. International Journal of Marine Energy 2015;9:20-40.

Newman JN. Wave effects on deformable bodies. Applied Ocean Research 1994;16(1):47-59. 
Ricci P, Saulnier J, Falcao A. Point-absorber arrays: a configuration study off the Portuguese west-coast. In: Proceedings of the 7th European Wave Tidal Energy Conference. Porto (Portugal); 2007. .

Taghipour R, Moan T. Efficient Frequency-Domain Analysis of Dynamic Response for the Multi-Body Wave Energy Converter in Multi-Directional Waves. Proceedings of the Eighteenth (2008) International Offshore and Polar Engineering Conference 2008;8:357-65.

Weller S, Stallard T, Stansby P. Experimental measurements of irregular wave interaction factors in closely spaced arrays. IET Renewable Power Generation 2010;4(6):628. 